\title{
Investigation of The Physical and Functional Needs in Adult Cancer Patients Consulted to Physiotherapy and Rehabilitation
}

\author{
Vesile Yıldız KABAK, Neslihan TAŞ, Yasin EKINCi, Songül Atasavun UYSAL, Tülin DÜGER \\ Department of Physical Therapy and Rehabilitation, Hacettepe University Faculty of Health Sciences, Ankara-Turkey
}

\begin{abstract}
OBJECTIVE
The purpose of this study was to investigate the physical and functional needs of hospitalized cancer patients consulted to physiotherapy and rehabilitation services.

\section{METHODS}

Total of 176 patients with various cancer diagnoses who were treated at Hacettepe University Oncology Hospital and were consulted to physiotherapy and rehabilitation were included in this retrospective study. Patient data regarding diagnosis, metastasis condition, and treatment types, as well as rehabilitation needs such as performance of daily life activities, physical function deficiencies, and sensoryperception problems were evaluated and recorded by physiotherapists.
\end{abstract}

\section{RESULTS}

Average age of the 87 male and 89 female patients included in this study was $56.25 \pm 14.53$ years. According to evaluations of performance of daily life activities, $137(77.8 \%)$ of the participants had difficulty with mobility, 132 (75\%) had difficulty with transfer activities, and $106(60.2 \%)$ had difficulty using the bathroom. It was observed that 162 (92\%) of the patients experienced deconditioning, $150(85.2 \%)$ had fatigue, and 149 (84.7\%) had balance problems. In addition, 42 (24.4\%) of the patients had sensory problems.

\section{CONCLUSION}

It is important to point out that cancer patients have various forms of rehabilitation needs, including functional deficiency, dependency in daily life activities, and sensory-perception-cognitive problems.

Keywords: Cancer; physiotherapy; rehabilitation.

Copyright $\odot$ 2016, Turkish Society for Radiation Oncology

\section{Introduction}

As the survival from cancer increases in the world and in our country, the chronic conditions caused by the disease have gained importance, making rehabilitation programs an important part of the treatment in order to maximize the functional level to the highest and to increase the quality of life.[1]
After being diagnosed with cancer, patients, families and caregivers make great efforts to cope up with the problems that emerge due to the disease and treatments. Patients' performance in their daily life, their ability to continue their profession or education, and their participation in family and social activities are adversely affected due to the progressive nature of the 
disease, as well as the side effects of applications such as chemotherapy and radiotherapy.[2]

In 1978, for the first time, Lehman et al. investigated the rehabilitation needs of 800 cancer patients and reported that their most prominent problems were the ones related to psychological stress, pain, muscle weakness, daily living activities, ambulance and family support.[3]

Emotional support, pain, difficulties in daily living activities, and mobility problems were reported by DePompolo in 1994; standing up from a chair, using the toilet, showering, walking and climbing the stairs were amongst the problems reported by Sabers in 1999; and sleeping disorders, pain, fatigue and anxiety were the problems stated by Whelan in 1997.[4-6]

In 1998, however, Van Harten pointed out that rehabilitation problems included not only physical function adversities but also psychosocial and cognitive function difficulties, and that several of these problems can be seen together.[7]

In fact, oncologic rehabilitation differs from rehabilitation programs for other diseases in some aspects. Here, physiotherapists treat their cancer patients with more flexible and differentiated treatment programs that take into account changes in their conditions resulting from both the disease and treatments. For example, daily exercise program varies depending on the level of fatigue and factors affecting it (blood table, chemotherapy session, etc.). Hence, it is important that the cancer patients are evaluated in detail, their needs are identified and any changes in their conditions are detected immediately.

Taking these into account, the purpose of our study was to determine physiotherapy and rehabilitation needs of cancer patients who are hospitalized due to complications or some treatment procedures, and to help physiotherapists to establish an appropriate physiotherapy and rehabilitation program that meets all the needs of their cancer patients.

\section{Material and Methods}

Our study included cancer patients hospitalized at Hacettepe University Oncology Hospital between 2011 and 2015. For all participants physiotherapy and rehabilitation consultation was requested by the oncologist. In this retrospective, single-center, crosssectional study, adult patients with different cancer diagnoses were included. Ethics committee approval
Table 1 Evaluation of physical functions

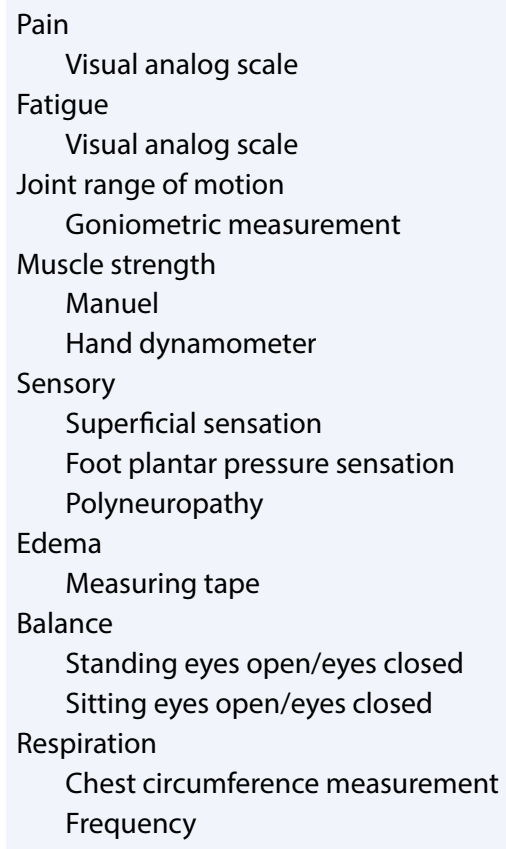

for this study was obtained from Hacettepe University, Non-Interventional Ethics Committee, No. GO 16/158-30.

Minimum one week of hospitalization, ability to communicate, being in the II-IV stage of the illness, and being in the age range of 18-65 years were the inclusion criteria. Patients who did not want to participate in the physiotherapy and rehabilitation program were excluded from the study.

Medical records of the participants were precisely checked and their physiotherapy-rehabilitation needs were evaluated in accordance with their diagnosis, anamnesis and major complaints. Along with recording the patients' diagnoses, demographic data, metastatic status, and their treatment, the participants' daily life activities (such as transfer, mobility, self-care and dressing), physical functional deficits (such as pain, fatigue, deconditioning, and balance problems) and their sensory-perceptual problems (such as sensory loss, polyneuropathy, cognitive and communication problems) were also evaluated and recorded by physiotherapists. The evaluation of physical functions is shown in Table 1.

\section{Statistical Analysis}

SPSS 10.0 package program was used for the analysis. Measurable data is expressed as arithmetic mean, \pm standard deviation. 


\section{Results}

176 cancer patients were included in the study, out of whom 89 (50.6\%) were female and 87 (49.4\%) were male; the average age was $56.25 \pm 14.536$ years.

Distribution of diagnosis of the individuals, which is shown in Table 2, reveals that lymphoma (20.5\%), breast cancer $(15.3 \%)$ and multiple myeloma (10.8\%) were the most common diagnosis.

Reasons for admission to the hospital are shown in Table 3, according to which patients were fre-

\begin{tabular}{lcc}
\hline Table 2 Diagnosis distributions & & \\
\hline Cancer type & $\mathbf{n}$ & \% \\
\hline Lymphoma & 36 & 20.5 \\
Breast cancer & 27 & 15.3 \\
Multiple myeloma & 19 & 10.8 \\
Lung cancer & 16 & 9.1 \\
Leukemia & 13 & 7.4 \\
Brain tumors & 13 & 7.4 \\
Colon cancer & 7 & 4.0 \\
Gastric cancer & 7 & 4.0 \\
Pancreatic cancer & 7 & 4.0 \\
Kidney cancer & 6 & 3.4 \\
Ovarium cancer & 4 & 2.3 \\
Liver cancer & 3 & 1.7 \\
Prostate cancer & 3 & 1.7 \\
Nasopharyngeal cancer & 2 & 1.1 \\
Larynx cancer & 2 & 1.1 \\
Endometrium cancer & 2 & 1.1 \\
Duodenum cancer & 2 & 1.1 \\
Adenoma & 1 & 0.6 \\
Bladder cancer & 1 & 0.6 \\
Esophageal cancer & 1 & 0.6 \\
Uterus cancer & 1 & 0.6 \\
Thymoma & 1 & 0.6 \\
\hline
\end{tabular}

\section{Table 3 Reasons of hospitalization}

\begin{tabular}{lcc} 
& $\mathbf{n}$ & $\%$ \\
\hline Diagnostic and therapeutic purposes & 45 & 25.5 \\
Deterioration in general health status & 36 & 20.4 \\
Inability to walk and/or loss of force & 30 & 17 \\
Respiratory problems & 16 & 9 \\
Pain & 13 & 7.3 \\
Infection & 12 & 6.8 \\
Cytopenia & 7 & 3.9 \\
Palliative care & 6 & 3.4 \\
Fatigue & 4 & 2.2 \\
Other & 7 & 3.9 \\
\hline
\end{tabular}

quently admitted to the hospital for diagnostic and/ or therapeutic purposes. Metastasis was recorded in $100(58.5 \%)$ of the individuals. Chemotherapy was applied in 49 (29.7\%), radiotherapy in $11(6.7 \%)$, radiotherapy and chemotherapy in 13 (7.9\%), and other treatments in $92(55.8 \%)$ of the participants. The distribution of patients according to their treatment procedures is given in Table 4 .

When individuals' physiotherapy and rehabilitation needs were assessed, it was determined that deconditioning, fatigue, balance problems, transfer and mobility difficulties were the most common and primary ones. In DLA, it was observed that patients had the most problems with mobility and transfers. Physiotherapy and rehabilitation needs of patients are categorized and their distributions are shown in Table 5, 6 and 7.

Table 4 Treatment status of the patients

\begin{tabular}{lcc} 
Ongoing treatments & $\mathbf{n}$ & $\%$ \\
\hline Chemotherapy & 49 & 29.7 \\
Radiotherapy & 11 & 6.7 \\
Chemotherapy and radiotherapy & 13 & 7.9 \\
Other & 92 & 55.8 \\
\hline
\end{tabular}

Table 5 Needs in daily living activities

\begin{tabular}{lcc} 
Needs in daily living activities & $\mathbf{n}$ & \% \\
\hline Mobility & 137 & 77.8 \\
Transfer & 132 & 75.0 \\
Using the bathroom & 106 & 60.2 \\
Dressing & 75 & 42.6 \\
Self care & 63 & 35.8 \\
Eating & 49 & 27.8
\end{tabular}

Table 6 Requirements for physical problems

\begin{tabular}{lcc} 
Physical problems/needs & $\mathbf{n}$ & \% \\
\hline Deconditioning & 162 & 92.0 \\
Fatigue & 150 & 85.2 \\
Balance problems & 149 & 84.7 \\
Pain & 113 & 64.2 \\
Edema & 58 & 33.0 \\
Limitation & 49 & 27.8 \\
Respiratory problems & 49 & 27.8 \\
Swallowing problems & 12 & 6.8 \\
Decubitus ulcers & 9 & 5.1 \\
Lymphedema & 7 & 4.0 \\
\hline
\end{tabular}


Table 7 Sensory, perception and cognitive problems

\begin{tabular}{lcc} 
Sensory-perception problems & n & \% \\
\hline Sensory problems & 63 & 35.8 \\
Cognitive problems & 20 & 11.4 \\
Communication problems & 20 & 11.4
\end{tabular}

\section{Discussion}

Results of our retrospective study, where cancer patients who were hospitalized due to complications or some treatment applications were evaluated in terms of their physiotherapy and rehabilitation needs revealed that daily living activities such as mobility, transfer and using the bathroom, as well as physical problems such as deconditioning, fatigue, balance problems, and pain were the most common complaints of the patients. Furthermore, polyneuropathy was found to be the most common sensory problem.

According to the results of the study in Switzerland by Ture et al. out of all cancer patients who were rehabilitated, the ones with digestive system tumors were the first, the patients with thoracic tumors were the second, and the ones with breast cancer were the third group who had benefitted from the rehabilitation program the most. [8] Moreover, male patients were reported to be less rehabilitated than female patients.

In the present study, diagnosis distribution of 176 cancer patients who required physiotherapy and rehabilitation consultation was reported in the following order: lymphoma, breast cancer, multiple myeloma, lung cancer, leukemia, and brain tumors. In our study, the need for rehabilitation was found to be equally important for both male and female patients. Metastasis was recorded in $58.5 \%$ of the patients participating in the study. This indicates that the diagnosis distributions of patients who require physiotherapy and rehabilitation consultation vary significantly and their illness is in an advanced stage.

Mavsas et al. investigated functional disorders and rehabilitation needs of 55 patients in the medical oncology unit and highlighted many previously unknown rehabilitation requirements of the patients; the most primary one of which was deconditioning.[1]

Aras et al. investigated rehabilitation needs of 300 in-patients of the oncology hospital who did not apparently require any physiotherapy and rehabilitation. [9] Results of the study showed that 245 (81.7\%) of the participants were actually in need of rehabilitation mostly due to fatigue, deconditioning, difficulties in daily living activities, ambulation problems, and pain. In addition, they also stated that joint contracture and lymphedema in patients with breast cancer, transfer problems in patients with lung cancer, and joint contractures in patients with bone cancers are more frequent. It was also reported that out of 245 patients who were identified as the ones in need of rehabilitation, only 2 were consulted to physiotherapy and rehabilitation unit.

In their study examining functional impairments and rehabilitation needs of non-operated lung cancer patients, Bayly et al. reported that fatigue, respiratory distress and pain are the most important issues to be considered in the rehabilitation program.[10]

In our study, evaluation of the patients' daily living activities showed the loss of independency in $77.8 \%$ of the patients in terms of mobility, in $75 \%$ in terms of transfer activities, and in $60 \%$ in terms of using the bathroom. In our patient group, for which physiotherapy and rehabilitation needs were identified by the oncologist, it was noticed that difficulties in mobility and transfer activities were very common, and that elevated levels of dependency during daily living activities was the reason for requesting physiotherapy and rehabilitation consultation.

Rehabilitation needs of the patients in accordance with their physical problems were determined as: $92 \%$ deconditioning, $85.2 \%$ fatigue, $84.7 \%$ balance disorders, and $64.2 \%$ pain. Similar to the literature, we also concluded that deconditioning and fatigue were the most prominent problems of the patients within the physiotherapy and rehabilitation program. Moreover, edema/lymphedema, joint motion limitations, respiratory problems and swallowing problems were also determined depending on the diagnosis and involvement zone. Hence, deconditioning and fatigue leading to deficits in balance, mobility and transfers were among the first clues that would cause physicians to consult physiotherapy and rehabilitation unit. Patients suffering excessive fatigue and ambulatory loss are seen to be the primary candidates for physiotherapy and rehabilitation consultation.

Gültekin et al. examined health care expectations of 40 lung patients.[11] According to the results, patients expect physicians to "cease the pain", nurses to "relieve pain and discomfort", psychologists to "reduce their sadness and grief", dietitians to "prevent weight loss", physiotherapists to "eliminate muscle weakness", and social workers to "help them maintain good relationships with others". This suggests that patients need a multidisciplinary team to tackle and cope with their problems. 
The rehabilitation needs we have shown in our work show that physiotherapists, as an important member of the multidisciplinary team, are needed for the treatment and follow-up of cancer patients.

The researchers also concluded that functional malfunctions of cancer patients are not adequately and precisely identified, appropriate and timely physiotherapy and rehabilitation consultations are generally underestimated, and family members are not trained and informed about rehabilitation needs of the patients. In addition, in the study investigating and comparing the needs of cancer patients in admission to and discharge from the hospital; it was emphasized that the rehabilitation needs of the patients were continued at discharge and that they should be followed up with home programs.[1]

\section{Conclusion}

The present study showed that high levels of dependency in daily living activities and major problems in physical functions identified in cancer patients with physiotherapy and rehabilitation consultation is an indication of the lack of knowledge of health professionals in this area. Problems identified in cancer patients are related to each other and they generally continue for a certain period of time; that's why it is believed that training patients and their families, as well as health professionals on physiotherapy and rehabilitation in cancer can be a solution to overcome these deficiencies.

\section{Disclosure Statement}

The authors declare no conflicts of interest.

\section{References}

1. Movsas SB, Chang VT, Tunkel RS, Shah VV, Ryan LS, Millis SR. Rehabilitation needs of an inpatient medical oncology unit. Arch Phys Med Rehabil 2003;84(11):1642-6. Crossret
2. O’Toole DM, Golden AM. Evaluating cancer patients for rehabilitation potential. West $\mathrm{J}$ Med 1991;155(4):384-7.

3. Lehmann JF, DeLisa JA, Warren CG, deLateur BJ, Bryant PL, Nicholson CG. Cancer rehabilitation: assessment of need, development, and evaluation of a model of care. Arch Phys Med Rehabil 1978;59(9):410-9.

4. DePompolo RW. Development and administration of a cancer rehabilitation program. In Garden FH, Grabois, editors. Cancer Rehabilitation State of the Art Reviews. Vol 8. Philadelphia: Hanley\&Belfuß; 1994. p. 419.

5. Sabers SR, Kokal JE, Girardi JC, Philpott CL, Basford JR, Therneau TM, et al. Evaluation of consultation-based rehabilitation for hospitalized cancer patients with functional impairment. Mayo Clin Proc 1999;74(9):855-61. Crossret

6. Whelan TJ, Mohide EA, Willan AR, Arnold A, Tew $\mathrm{M}$, Sellick S, et al. The supportive care needs of newly diagnosed cancer patients attending a regional cancer center. Cancer 1997;80(8):1518-24. Crossre]

7. van Harten $W H$, van Noort O, Warmerdam R, Hendricks $\mathrm{H}$, Seidel E. Assessment of rehabilitation needs in cancer patients. Int J Rehabil Res 1998;21(3):24757. Crossret

8. Ture M, Barth J, Angst F, Aeschlimann A, Schnyder U, Zerkiebel N, et al. Use of inpatient rehabilitation for cancer patients in Switzerland: who undergoes cancer rehabilitation? Swiss Med Wkly 2015;145:w14214.

9. Aras M, Ünsal Delialioğlu S, Atalay N, Taflan-Selçuk S. Rehabilitatıon Needs of Patients with Cancer. Türk Fiz Tip Rehab Derg 2009;55:25-9.

10. Bayly JL, Lloyd-Williams M. Identifying functional impairment and rehabilitation needs in patients newly diagnosed with inoperable lung cancer: a structured literature review. Support Care Cancer 2016;24(5):2359-79. Crossre

11. Gültekin Z, Pınar G, Pınar T, Kızıltan G, Doğan N, Algier L, et al. Health-Related Quality of Life and Health Care Services Expectations of The Patients with Lung Cancer. Uluslararası Hemotoloji Onkoloji Dergisi 2008;2(18);99-106. 